\title{
Identification of an MSI-H Tumor-Specific Cytotoxic T Cell Epitope Generated by the (-1) Frame of U79260(FTO)
}

\author{
Michael Linnebacher, Anne Wienck, Inga Boeck, and Ernst Klar \\ Section of Molecular Oncology and Immunotherapy, Department of General, Thoracic, Vascular and Transplant Surgery, \\ University of Rostock, Schillingallee 35, 18057 Rostock, Germany \\ Correspondence should be addressed to Michael Linnebacher, michael.linnebacher@med.uni-rostock.de
}

Received 17 November 2009; Accepted 14 January 2010

Academic Editor: Zhengguo Xiao

Copyright (๑) 2010 Michael Linnebacher et al. This is an open access article distributed under the Creative Commons Attribution License, which permits unrestricted use, distribution, and reproduction in any medium, provided the original work is properly cited.

\begin{abstract}
Microsatellite instability (MSI-H) induced by defects of the DNA mismatch repair system results in insertion or deletion of single nucleotides at short repetitive DNA sequences. About $15 \%$ of sporadic and approximately $90 \%$ of hereditary nonpolyposis colorectal cancers display MSI-H. When affecting coding regions, MSI-H results in frameshift mutations and expression of corresponding frameshift peptides (FSPs). Functional tumor promoting relevance has been demonstrated for a growing number of genes frequently hit by MSI-H. Contrary, immune reactions against FSPs are involved in the immune surveillance of MSI-H cancers. Here, we provide conclusive data that the (-1) frame of U79260(FTO) encodes an HLA-A0201-restricted cytotoxic T cell epitope (FSP11; TLSPGWSAV). T cells specific for FSP11 efficiently recognized HLA-A0201 ${ }^{\text {(pos) }}$ tumor cells harboring the mutated reading frame. Considering the exceptionally high mutation rate of U79260(FTO) in MSI-H colorectal carcinoma (81.8\%), this recommends that FSP11 be a component of future vaccines.
\end{abstract}

\section{Introduction}

Loss of genomic stability is the key molecular step in cancer formation [1]. Virtually all colon cancers display either chromosome instability (CIN) or microsatellite instability (MSI-H) indicating that genomic instability is central to colon cancer formation. In hereditary colorectal cancer syndromes, germline forms of key genetic defects have been identified whose somatic occurrences drive the emergence of sporadic colon tumors [2]. Inactivation of the DNA mismatch repair system (MMR) due to germline defects causes the hereditary nonpolyposis colon cancer syndrome (HNPCC). Somatic inactivation of the MMR system due to methylation of the promoter of hMLH1 additionally gives rise to about $15 \%$ of sporadic colon tumors [3]. The resulting colon cancers display the phenotype of MSI-H recognized as length variations in short repetitive DNA sequences called microsatellites.

Instability of microsatellites in coding regions results in frameshift mutations preceding expression of C-terminally truncated proteins with attached neopeptide tails. Several genes known to play important roles in colorectal carcinogenesis as, for example, TGFbetaRII, Bax, AIM2, and TCF4 are targeted by this mutational mechanism, leading to the hypothesis that these truncated gene products have an impaired function and might thereby contribute to MSI-Hdriven carcinogenesis [4-7]. Several investigators have aimed to identify other commonly mutated coding microsatellites (cMS) in MSI-H tumors, and as a result of these efforts, numerous candidates have been identified with varying mutation frequencies [8-10].

In any case, following proteasomal degradation, parts of these novel proteins might be presented as immunogenic neopeptides in the context of MHC molecules at the surface of MSI-H cancer cells, thereby allowing for recognition of frameshift-derived nonself by effector cells of the immune system. Several features of MSI-H tumors such as enhanced lymphocytic infiltration, Crohn's-like reactions, high prevalence of activated intraepithelial cytotoxic T lymphocytes, and increased neoplastic cell apoptosis argue for the presence of an antitumoral immune reaction directed against potent tumor rejection antigens [11-14]. 
Thus far, we and others demonstrated the immunogenicity of $(-1)$ frameshift mutations in coding microsatellites of TGFbRII, OGT, and Caspase-5 [15-19]. These data established cMSI-induced frameshift-derived neopeptides as a novel class of tumor-associated antigens likely to represent main targets of MSI-H tumor-specific immune reactions. In support of this hypothesis, Tougeron et al. demonstrated a close correlation between tumor-infiltrating lymphocytes and the expression of frameshift mutations in MSI-H CRC tumors [20]. Finally, we were able to show that $\mathrm{T}$ cells specific for MSI-H-induced frameshift mutation can regularly be found in the peripheral blood of HNPCC mutation carriers [21]. This latter finding was independent from the actual presence of a diagnosable tumor. Considering the high specificity of frameshift peptides for MSI-H tumor cells or precursors thereof, they are most likely very interesting targets for usage in specific antitumoral immunotherapy. So far, the number of identified frameshift-derived $\mathrm{T}$ cell epitopes is very limited. However, for creation of successful vaccines it seems reasonable to combine several epitopes derived from different antigens. Therefore, it is imperative to identify more such peptide sequences able to induce a potent $\mathrm{T}$ cell response.

In the present study we characterized the immunogenic potential of the HLA-A0201-restricted frameshift peptide (FSP)11 (TLSPGWSAV) derived from the fat mass and obesity-associated gene U79260(FTO). This gene is located on chromosome 16q12.2 (gi:1710215). The function of the gene product is still unknown, but as the name implies, it seems to be involved in energy metabolism (reviewed in [22]). The mRNA shows a very high mutation rate of $81.8 \%$ in colon carcinomas and up to $100 \%$ in established colorectal carcinoma cell cultures as opposed to $6.7 \%$ in stomach and $41.7 \%$ in endometrial carcinomas, indicating a high specificity for colorectal cancer cells (summarized in [23]).

\section{Material and Methods}

2.1. Tumor Cell Lines and Peptides. All tumor cell lines used in the present study (Colo60H, HLA-0201 ${ }^{\text {(pos) } / U 79260(F T O) ~}$ (-3); HCT116, HLA-0201 ${ }^{\text {(pos) } / U 79260(F T O) ~(-4) ; ~ S W 480: ~}$ HLA-0201 ${ }^{\text {(pos) } / U 79260(F T O) ~(w t) ; ~ a n d ~ K M 12: ~ H L A-~}$ $0201^{(\mathrm{neg})} / U 79260$ (FTO) $^{(-4))}$ were obtained from ATCC, CLS (Heidelberg, Germany), or from the German Cancer Research Center (DKFZ). They were grown in DMEM/Ham's F-12 (1 : 1) medium supplemented with 10\% fetal bovine serum (FCS), $2 \mathrm{mmol} / \mathrm{l}$ glutamine, and antibiotics unless stated otherwise. The HLA-A0201 expressing cell line T2 $(174 \times$ CEM.T2 hybridoma, TAP1, and TAP2 deficient) was used in some experiments. All media and supplements were obtained from PAA (Coelbe, Germany) unless stated otherwise.

Peptide FSP11 derived from the (-1)-mutated form of U79260(FTO) (TLSPGWSAV; gi:1710215) and control peptide (MP: GILGLVFVTL) were obtained from the Peptide
Synthesis Facility of the DKFZ. They were dissolved in DMSO $(5 \mathrm{mg} / \mathrm{ml})$ and further diluted in PBS $(500 \mu \mathrm{g} / \mathrm{mL})$.

2.2. Generation of CD40-Activated B Cells and Peptide-Specific Stimulation of T Cells. Peripheral blood was obtained from a healthy HLA-0201(pos) donor after informed consent in written form was obtained. The experiments were approved by the responsible Ethical Committee. B and $\mathrm{T}$ cells were purified as described in detail before [15]. B cells were stimulated via NIH/3T3 fibroblasts stably expressing human CD154 (t-CD 154). Lethally irradiated (60 Gy) t-CD154 were plated on 6 -well plates $\left(0.4 \times 10^{5}\right.$ cells/well $)$ and cultured overnight. After rinsing with PBS, the B cells were added $(1 \times$ $10^{6}$ cells/well) in medium containing $10 \%$ FCS, $2 \mathrm{mmol} / \mathrm{l}$ glutamine, supplements ( $1: 100)$, and IL-4 (2 ng/mL). The outgrowing B cells (CD40 Bs) were restimulated every 3-4 days.

CD40 Bs were irradiated (30 Gy), incubated with peptide $(5 \mu \mathrm{g} / \mathrm{mL})$ in serum-free IMDM for 1 hour at $37^{\circ} \mathrm{C}$, and washed to remove excess of peptide. Then the CD40 Bs were added to purified $\mathrm{CD}^{+}$autologous $\mathrm{T}$ cells at a ratio of 4 : 1 ( T:CD40 Bs) in T cell medium (IMDM containing 10\% FCS, $2 \mathrm{mmol} / \mathrm{l}$ glutamine, and antibiotics), supplements (1 : 100), and IL-7 (10 IU/mL). T cells were restimulated every 7 days; IL-7 was replaced by IL-2 (100 IU/mL) on day 28.

2.3. Cloning of FSP11-Specific Cytotoxic T Lymphocytes (CTLs). Cloning of FSP11-specific T cells was performed by limiting dilution of the $\mathrm{T}$ cell bulk culture. Briefly, 0.7 cells/well were plated in V-bottomed 96-well plates. Lethally irradiated (30 Gy) FSP11-loaded CD40 Bs of the autologous donor $\left(2 \times 10^{4} /\right.$ well $)$ were added to the diluted $\mathrm{T}$ cells in a final volume of $200 \mu \mathrm{l}$ IMDM containing $10 \%$ FCS, supplements $(1: 100)$ and IL-2 (100 IU/mL). This treatment was repeated weekly for restimulation. After 4 weeks, outgrowing clones were screened for specific cytotoxic activity in IFN- $\gamma$-ELISpot and subsequently in FACSotox Assays.

2.4. IFN- $\gamma$-ELISpot Assay. ELISpot assays were performed using nitrocellulose-96-well plates (Multiscreen; Millipore, Bedford, MA) covered with mouse antihuman IFN- $\gamma$ mAb (Mabtech, Nach, Sweden) and blocked with FCS-containing medium. T cells $\left(1 \times 10^{3}\right)$ were plated in sixplicate with $1 \times 10^{4} \mathrm{~T} 2$ or CD40 Bs as targets in $200 \mu \mathrm{IMDM}$ with $10 \%$ FCS. Peptides were added at a final concentration of $10 \mu \mathrm{g} / \mathrm{mL}$. After incubation for 16 hours at $37^{\circ} \mathrm{C}$, plates were washed and incubated with biotinylated rabbit-antihuman IFN $\gamma$ mAb for 4 hours, washed again and incubated with streptavidin-coupled alkaline phosphatase followed by a final wash. For spot detection the plate was incubated with NBT/BCIP (Promega, Madison, WI). The reaction was stopped after 45-60 minutes by washing the plate with water. The spots were counted after drying. The deduced frequency of peptide-specific $\mathrm{T}$ cells was calculated by substracting the mean number of spots in the neopeptide control from the mean number of IFN- $\gamma$ spots in the peptide-stimulated 
sample. Occasionally, resulting values were negative; those were scored as zero.

To confirm HLA-A0201 restriction of the FSP11-specific T cells, antibody-blocking ELISpots were performed. FSP11pulsed CD40 Bs were incubated with the monoclonal antibodies anti-HLA-A2 (clone BB7.2) and pan-anti-MHCI (clone W6/32) and T cells with anti-CD3 (clone OKT3) and anti-CD8 (clone OKT8). Irrelevant IgG2 antibody served as a control (Immunotools, Friesoythe, Germany).

2.5. FACSotox Cytotoxicity Assay. Target cells $\left(1 \times 10^{6}\right.$; CD40 Bs, T2, tumor cells) were stained with $5 \mu \mathrm{M}$ CFSE in $1 \mathrm{~mL}$ of medium followed by an incubation at $37^{\circ} \mathrm{C}$ for 45 minutes. The cells were washed twice and resuspended in $1 \mathrm{ml}$ medium followed by 30 minutes incubation at $37^{\circ} \mathrm{C}$. For peptide loading, target cells were incubated with peptide $(5 \mu \mathrm{g} / \mathrm{mL})$ in medium without FCS for one hour at $37^{\circ} \mathrm{C}$. CD40 Bs, T2, and tumor cells were seeded into 96-well plates (10 000 cells/well). $T$ cells were added at different effector to target $(\mathrm{E}: \mathrm{T})$ cell ratios $(1: 5,1: 10,1: 20)$. For estimation of background, wells containing target cells were run without the addition of $\mathrm{T}$ cells. The plates were incubated at $37^{\circ} \mathrm{C}$ for 6 and 12 hours before assessment of cytotoxicity by flow cytometry. Prior to analysis, $15 \mu \mathrm{g} / \mathrm{mL}$ propidium iodide was added as a live/dead stain. The percentage of specific lysis was calculated as follows:

cytotoxicity $(\%)$ : $[100 \% \times$ dead target cells in experimental sample/(dead target cells in experimental sample + live target cells in experimental sample) $]-[100 \% \times$ dead target cells in target cell control/(dead target cells in target cell control + live target cells in target cell control)].

To further confirm peptide specificity, unstained CD40 Bs loaded with FSP11 or an irrelevant peptide, respectively, were added into some experiments. The ratio of unstained to stained targets was $20: 1$.

2.6. Flow Cytometric Analyses. In order to characterize $\mathrm{T}$ cell surface marker expression $3 \times 10^{5} \mathrm{~T}$, cells were washed in PBS and stained with the following directly FITC or PE labeled antibodies: CD3 (clone UCHT-1), CD8 (clone MEM31), CD45RO (clone UCHL1), CD50 (clone MEM-171), CD62L (clone LT-TD180), and CD102 (clone SM223F) for 30 minutes on ice. Cells were then washed twice in PBS and resuspended in PBS containing 1\% paraformaldehyde.

Cells stained with isotype controls were used as negative controls. Antibodies were obtained from Immunotools or Acris (Herford, Germany). Intensities were analyzed with a FACScalibur flow cytometer (BD Biosciences).

2.7. Fragment Length Analysis. Genomic DNA was isolated from cell lines according to standard protocols. PCR was performed as described [23] . PCR primers for U79260(FTO) were sense TTTgTTATATCCCATTAggTgCC and antisense AgCCTggTgACAgAgTgAgAC yielding a PCR product of about 130 nucleotides in length. Products were separated on an ABI3100 device (Perkin Elmer, Waltham, MA). As wt control, gDNA from PBMCs of a healthy donor was included into the analysis.
2.8. Statistics. All values are expressed as mean (SEM). Student's $t$-test was used to evaluate the differences between two measurements. The criterion for significance was taken to be $P=.05$ or lower.

\section{Results and Discussion}

U79260(FTO) is likely to be involved in colorectal carcinogenesis as there is an obvious positive selection pressure towards high mutation rates in colon carcinomas (81.8\%) when compared to normal tissue and especially to other MSI-H tumor entities (mutation rates of $6.7 \%$ in stomach and $41.7 \%$ in endometrial carcinomas, in [8] and summarized in [23]).

So far, we and others have shown that peptides derived from frameshift mutations in genes coding for TGF-betaIIR $[15,16]$, O-GlcNac transferase (OGT) [18], and Caspase5 [19] gave rise to CTL capable to lyse MSI-H cancer cell lines expressing these frameshift mutations. Meanwhile, the immunogenic character of MSI-H-induced frameshift mutations has been corroborated. Saeterdahl et al. identified an MHC-class-II-restricted T helper epitope and Ishikawa et al. demonstrated the existence of specific antibodies directed against a frameshifted cMS target gene [17, 24].

Only recently, we analyzed peripheral $\mathrm{T}$ cell reactivity towards several FSPs of patients with present MSI-H colorectal carcinoma and of HNPCC mutation carriers without a history of tumor development [21]. A peptide mixture covering U79260(FTO) $(-1)$ was specifically recognized by peripheral $\mathrm{T}$ cells of $25 \%$ of patients (8/32) and of $31 \%$ of healthy mutation carriers (5/16). Additionally, reactivity could also be observed in two out of three in vitro expanded tumor infiltrating $\mathrm{T}$ cell populations derived from MSI-H tumor lesions [21]. However, formal proof of antitumoral (killing) activity of FSP-specific T cells was not provided in that study.

3.1. Generation of FSP11-Specific Cytotoxic T Cells. In order to analyze the immunogenic potential of the HLA-A0201restricted peptide FSP11 derived from a $(-1)$ mutation of a $\mathrm{T}(15)$ tract within the U79260(FTO) gene, we generated a $\mathrm{T}$ cell bulk culture from an HLA-0201(pos) donor. Previously, we could demonstrate that FSP11 was able to stabilize HLAA0201 molecules in a T2 stabilization assay, thus confirming HLA-A0201 binding properties [15]. As for antigenpresenting cells, we used CD40-activated B cells (CD40 Bs) from the autologous blood donor taking advantage of the CD40L culture system [15]. These CD40 Bs were loaded with FSP11 and used for repetitive stimulation of autologous T cells. After 28 days in culture the number of $\mathrm{T}$ cells had increased 5.6-fold. Stimulation was continued for more than 100 days and resulted in a T cell expansion exceeding 1000fold (data not shown).

Phenotypic characterization of stimulated cells by flow cytometric analysis confirmed that the bulk culture nearly exclusively contained $\mathrm{T}$ cells with cytotoxic potential (CD3: 99.9\% and CD8: $99.0 \%$ at day 77 of culture; see Table 1). A large part of the $\mathrm{T}$ cell population expressed the intercellular 
TABLE 1: Flow cytometric analysis of cell surface markers of FSP11specific T cells. Expression of several cell surface markers of the FSPspecific $T$ cell bulk culture was analyzed on day 91 of culture by FACS. Given is the percentage of positive cells. The corresponding isotype controls were always under $2.5 \%$.

\begin{tabular}{lc}
\hline Antigen & Positive cells (\%) \\
\hline CD3 & 99.9 \\
CD4 & 0.8 \\
CD8 & 99.0 \\
CD45RA & 27.0 \\
CD45RO & 60.4 \\
CD50 & 97.6 \\
CD54 & 61.5 \\
CD62L & 29.4 \\
CD102 & 56.6 \\
\hline
\end{tabular}

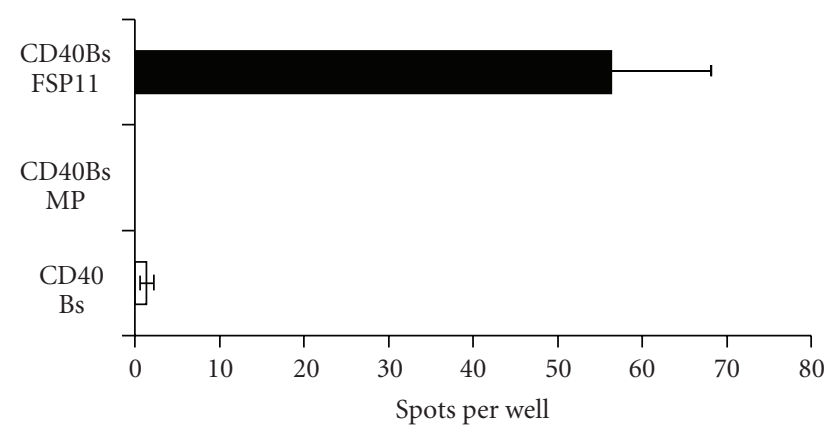

Figure 1: ELISpot analysis. The percentage of $\mathrm{T}$ cells secreting IFN- $\gamma$ in response to FSP11-loaded target cells was determined in a series of ELISpot experiments. An exemplary sample is shown. Reactivity against unloaded, irrelevant MP-peptide-loaded and FSP11-loaded CD40 Bs is displayed. Analysis was performed in triplicates with 1000 effector cells per well.

adhesion molecules CD50 (97.6\%), CD54 (61.5\%), and CD102 (56.6\%); a smaller fraction showed expression of the activation markers CD45RO (60.4\%) and CD62L (29.4\%; see Table 1). Considering the $27.0 \%$ expression of CD45RA, these data imply that the bulk culture contains both central and effector memory $\mathrm{T}$ cells.

Specificity of FSP11 recognition could readily be demonstrated in IFN- $\gamma$-ELISpot analysis. Here, the number of IFN$\gamma$-secreting $\mathrm{T}$ cells was determined upon stimulation with HLA-A0201-positive target cells loaded with cognate and control peptides, respectively. Approximately 5.5\% of the bulk T cells specifically secreted IFN- $\gamma$ in response to FSP11loaded autologous B cells as target cells (Figure 1). Similar results were also obtained using $\mathrm{T} 2$ cells as targets. These results imply FSP11 specificity of the T cell bulk culture. Additionally, the recognition can be attributed towards HLAA0201 as T2 cells do not express any other HLA allele.

3.2. FSP11-Specific and HLA-A0201-Restricted Recognition of Target Cells. Next, we analyzed the cytotoxic potential of the FSP11-specific T cell bulk. T2 as well as B cell target cells were

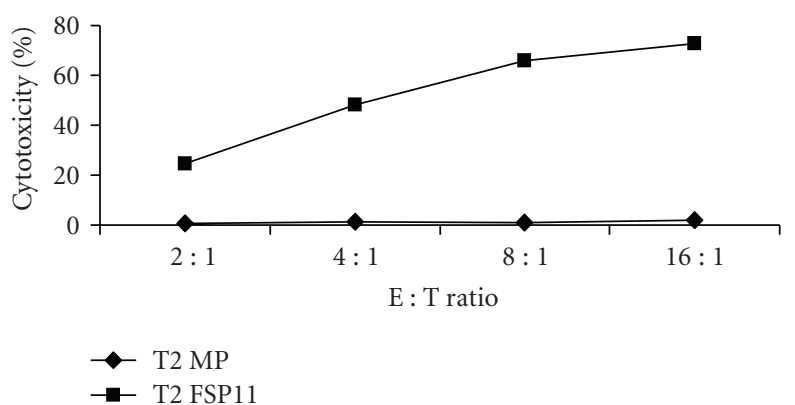

(a)

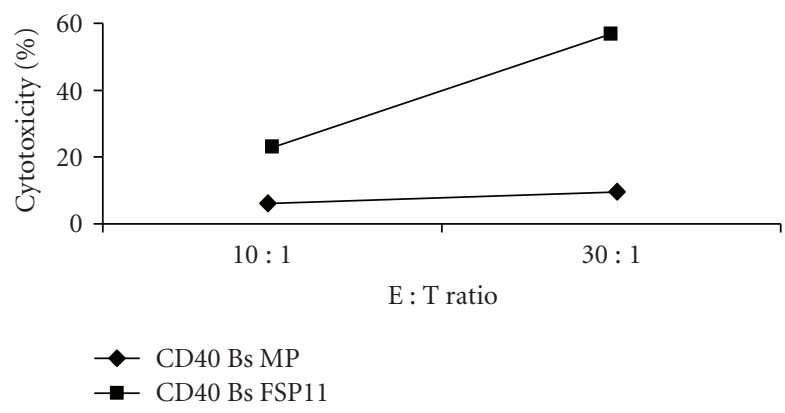

(b)

FIGURE 2: Analysis of cytotoxic potential of the FSP11-specific T cell bulk. FACSotox was performed using peptide-loaded (either FSP11 (square) or control peptide MP (diamond)) (a) T2 cells and (b) CD40 Bs as target cells. Effector bulk T cells were added at different effector to target cell ( $\mathrm{E}: \mathrm{T})$ ratios. A representative experiment of three is shown. Analysis was performed after a 6-hour incubation period.

selectively lysed when loaded with FSP11. Target cells loaded with an irrelevant peptide were not recognized (Figure 2).

To formally confirm FSP11 specificity and HLA-A0201 restriction, target kill inhibition experiments were performed by adding an excess of unstained CD40 Bs into these experiments. Lysis of stained and FSP11-loaded CD40 Bs was substantially inhibited by an excess of unstained target cells loaded with FSP11 (ratio of labeled targets: unlabeled targets $=1: 20$ ). In contrast, unstained CD40 Bs loaded with the irrelevant MP peptide had much less effect on target cell lysis (Figure 3). Moreover, the number of IFN- $\gamma$ secreting $\mathrm{T}$ cells in response towards FSP11 was significantly reduced by adding antibodies directed against pan MHC-class-I or against HLA-A0201 into ELISpot analysis (Figure 4).

In summary, these data reliably demonstrate specific recognition of FSP11 by the bulk $\mathrm{T}$ cells in the context of HLA-A0201. Additionally, antibodies directed towards cytotoxic T cells (CD3 and CD8) also significantly blocked the recognition (Figure 4).

3.3. Lysis of Colorectal Carcinoma Cells Endogenously Expressing U79260(FTO) (-1). Woerner et al. described that most MSI-H colorectal tumor cell lines $(6 / 11)$ carry $(-1)$ or (-4) mutations of U79260(FTO) [23]. To eliminate potential mistakes originating from expression of different mutations 


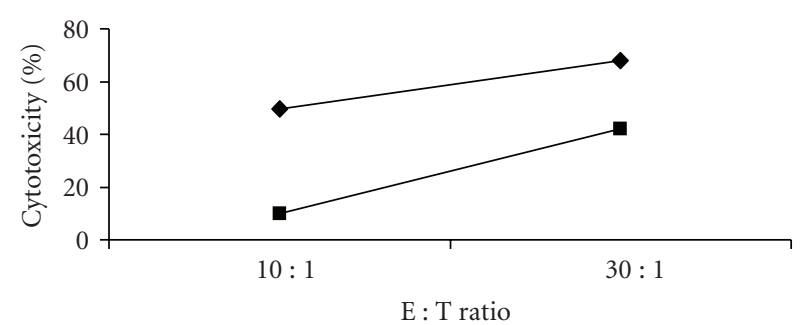

$\neg$ CD40 Bs MP

- CD40 Bs FSP11

FIgURE 3: Target inhibition. FSP11 specificity and HLA-A0201 restriction of target cell lysis of FSP11-specific T cells were tested at two different $\mathrm{E}: \mathrm{T}$ cell ratios $(10: 1,30: 1)$ in the presence of an excess of unlabeled target cells (CD40 Bs pulsed with either FSP11 (square) or control peptide MP (diamond)) at an unlabeled: labeled target cell ratio of $20: 1$ ). Displayed is the percentage of labeled and FSP11-loaded CD40 Bs killed by the FSP11-specific T cells. A representative experiment of three is shown. Analysis was performed after a 6-hour incubation period.

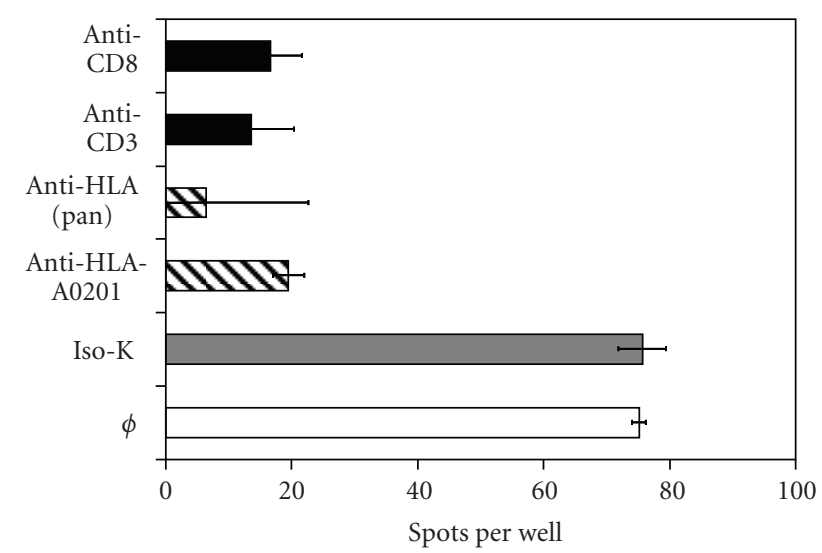

FIGURE 4: Antibody-blocking ELISpot analysis. FSP11 recognition of FSP11-specific T cells could be blocked by antibodies towards HLA(pan), HLA-A0201, CD3, and CD8 but not by IgG2 control antibody. The reactivity without the addition of antibodies is given as positive control. T2 cells loaded with FSP11 were used as targets. Either T2 cells or T cells were preincubated with antibodies towards HLA(pan) and HLA-A0201 (T2) or CD3 and CD8 (T cells) for one hour prior to setup of the ELISpot.

and wt forms of U79260(FTO) in different batches of MSI-H cell lines, the batches used in the current set of experiments were analyzed by fragment length analysis (data not shown).

Although specificity and cytotoxic potential of the bulk $\mathrm{T}$ cells have been shown, the most important feature in the context of antitumoral activity is the ability to recognize tumor cells expressing the underlying mutation endogenously. The FSP11 specific bulk culture specifically recognized the MSI-H, HLA-0201 (pos) colon carcinoma line HCT116, which endogenously expresses a $(-4)$-mutated form of $U 79260$ (FTO) corresponding to the $(-1)$ frame coding for FSP11 (Figure 5(a)). Contrary to that, there was neither significant recognition of the HLA-A0201 ${ }^{\text {(neg) }}$ cell line KM12, which expresses a (-4) version of U79260(FTO),

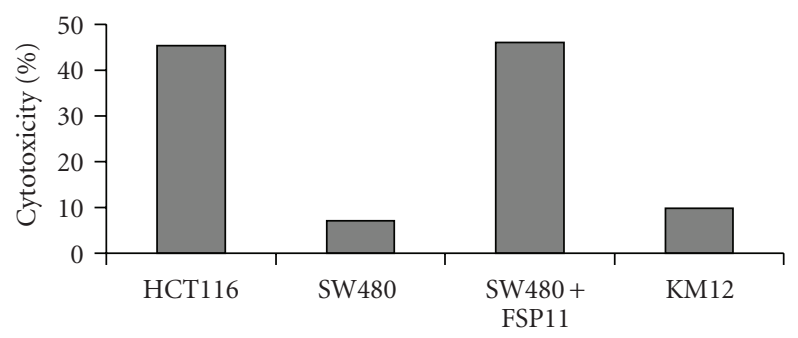

(a)

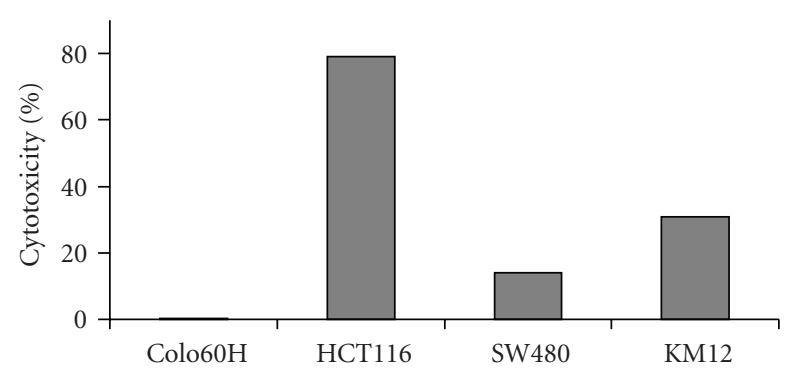

(b)

Figure 5: FSP11-specific $\mathrm{T}$ cells recognize tumor cell targets. $\mathrm{T}$ cell reactivity of the FSP11-specific (a) $\mathrm{T}$ cell bulk and (b) $\mathrm{T}$ cell clone C10/11 towards different tumor cell targets was tested at an effector to target (E : T) cell ratio of 20 : 1. Targets tested were Colo60H, HLA-0201(pos)/ U79260(FTO) (-3); HCT116, HLA-0201 ${ }^{\text {(pos) } / U 79260(F T O) ~(-4) ; ~ S W 480: ~ H L A-~}$ $0201^{\text {(pos) } / U 79260(F T O)}$ (wt) with and without addition of FSP11; and KM12: HLA-0201 (neg)/U79260(FTO) (-4). A representative experiment of three is shown. Analysis was performed after a 12hour incubation period.

nor of the HLA-A0201 ${ }^{\text {(pos) }}$ SW480 cells. However, SW480 is recognized when FSP11 is added exogenously (Figure 5(a)).

Comparable results were obtained using a CTL clone $(\mathrm{C} 10 / 11)$ generated from the FSP11 bulk $\mathrm{T}$ cell culture by limiting dilution. At an effector to target cell ratio of 5 : 1 , HCT116 cells (HLA-A0201 ${ }^{\text {(pos) }}$; U79260(FTO) $(-4)$ ) were effectively killed, whereas SW480 (HLA-A0201(pos); U79260(FTO) (wt)) and Colo60H (HLA-A0201 ${ }^{\text {(pos); }}$ U79260(FTO) (-3)) control cells were relatively unaffected (Figure 5(b)).

\section{Conclusions}

In the present study, we identify a novel MHC-class-Irestricted CTL epitope derived from U79260(FTO). The peptide FSP11 (TLSPGWSAV) sensitized peripheral CD8 ${ }^{+}$ $\mathrm{T}$ cells of a healthy donor for specific release of IFN- $\gamma$ and mediated recognition in the context of HLA-A0201. The obtained $\mathrm{T}$ cell cultures were able to selectively attack tumor cells expressing the underlying mutation together with HLA-A0201. Remarkably, these results additionally imply that U79260(FTO) must be expressed and translated into a truncated protein that is subsequently processed by the cellular proteasomal machinery and presented in the context of MHC molecules. 
Generally, MSI-H-induced FSPs represent an outstanding class of tumor-specific antigens that may prove suitable for future clinical applications. Beside immunotherapeutic interventions for patients suffering from an MSI-H tumor, this even opens up the remarkable possibility of prophylactic vaccinations for HNPCC mutation carriers.

\section{Abbreviations}

CD40 Bs: CD40-activated B cells

CTL: $\quad$ Cytotoxic T lymphocyte

ELISpot: Enzyme-linked immunospot

E : T ratio: Effector : target cell ratio

FACSotox: Fluorescence-activated cell-sorting-based cytotoxicity assay

FSP: $\quad$ Frameshift peptide

HLA: Human leucocyte antigen

MHC: $\quad$ Major histocompatibility complex

MMR: Mismatch repair system

MSI-H: (High level) Microsatellite instability.

\section{References}

[1] C. Lengauer, K. W. Kinzler, and B. Vogelstein, "Genetic instabilities in human cancers," Nature, vol. 396, no. 6712, pp. 643-649, 1998.

[2] K. W. Kinzler and B. Vogelstein, "Lessons from hereditary colorectal cancer," Cell, vol. 87, no. 2, pp. 159-170, 1996.

[3] S. N. Thibodeau, A. J. French, J. M. Cunningham, et al., "Microsatellite instability in colorectal cancer: different mutator phenotypes and the principal involvement of hMLH1," Cancer Research, vol. 58, no. 8, pp. 1713-1718, 1998.

[4] J. Wang, L. Sun, L. Myeroff, et al., "Demonstration that mutation of the type II transforming growth factor $\beta$ receptor inactivates its tumor suppressor activity in replication errorpositive colon carcinoma cells," Journal of Biological Chemistry, vol. 270, no. 37, pp. 22044-22049, 1995.

[5] Y. Shimizu, S. Ikeda, M. Fujimori, et al., "Frequent alterations in the Wnt signaling pathway in colorectal cancer with microsatellite instability," Genes Chromosomes and Cancer, vol. 33, no. 1, pp. 73-81, 2002.

[6] N. Rampino, H. Yamamoto, Y. Ionov, et al., "Somatic frameshift mutations in the BAX gene in colon cancers of the microsatellite mutator phenotype," Science, vol. 275, no. 5302, pp. 967-969, 1997.

[7] S. Dihlmann, M. Kloor, C. Fallsehr, and M. von Knebel Doeberitz, "Regulation of AKT1 expression by beta-catenin/ Tcf/Lef signaling in colorectal cancer cells," Carcinogenesis, vol. 26, no. 9, pp. 1503-1512, 2005.

[8] S. M. Woerner, M. Kloor, A. Mueller, et al., "Microsatellite instability of selective target genes in HNPCC-associated colon adenomas," Oncogene, vol. 24, no. 15, pp. 2525-2535, 2005.

[9] A. Duval, M. Reperant, and R. Hamelin, "Comparative analysis of mutation frequency of coding and non coding short mononucleotide repeats in mismatch repair deficient colorectal cancers," Oncogene, vol. 21, no. 52, pp. 8062-8066, 2002.

[10] N.-G. Kim, H. Rhee, L. S. Li, et al., "Identification of MARCKS, FLJ11383 and TAF1B as putative novel target genes in colorectal carcinomas with microsatellite instability," Oncogene, vol. 21, no. 33, pp. 5081-5087, 2002.
[11] S. M. Phillips, A. Banerjea, R. Feakins, S. R. Li, S. A. Bustin, and S. Dorudi, "Tumour-infiltrating lymphocytes in colorectal cancer with microsatellite instability are activated and cytotoxic," British Journal of Surgery, vol. 91, no. 4, pp. 469-475, 2004.

[12] T. C. Smyrk, P. Watson, K. Kaul, and H. T. Lynch, "Tumorinfiltrating lymphocytes are a marker for microsatellite instability in colorectal carcinoma," Cancer, vol. 91, no. 12, pp. 2417-2422, 2001.

[13] R. Dolcetti, A. Viel, C. Doglioni, et al., "High prevalence of activated intraepithelial cytotoxic $\mathrm{T}$ lymphocytes and increased neoplastic cell apoptosis in colorectal carcinomas with microsatellite instability," American Journal of Pathology, vol. 154, no. 6, pp. 1805-1813, 1999.

[14] H. T. Lynch and J. F. Lynch, "Genetics of colonic cancer," Digestion, vol. 59, no. 5, pp. 481-492, 1998.

[15] M. Linnebacher, J. Gebert, W. Rudy, et al., "Frameshift peptide-derived T-cell epitopes: a source of novel tumorspecific antigens," International Journal of Cancer, vol. 93, no. 1, pp. 6-11, 2001.

[16] I. Saeterdal, M. K. Gjertsen, P. Straten, J. A. Eriksen, and G. Gaudernack, "A TFG $\beta$ RII frameshift-mutation-derived CTL epitope recognised by HLA-A2-restricted CD8 ${ }^{+}$T cells," Cancer Immunology, Immunotherapy, vol. 50, no. 9, pp. 469476, 2001.

[17] I. Saeterdal, J. Bjørheim, K. Lislerud, et al., "Frameshiftmutation-derived peptides as tumor-specific antigens in inherited and spontaneous colorectal cancer," Proceedings of the National Academy of Sciences of the United States of America, vol. 98, no. 23, pp. 13255-13260, 2001.

[18] E. Ripberger, M. Linnebacher, Y. Schwitalle, J. Gebert, and M. Von Knebel Doeberitz, "Identification of an HLAA0201-restricted CTL epitope generated by a tumor-specific frameshift mutation in a coding microsatellite of the OGT gene," Journal of Clinical Immunology, vol. 23, no. 5, pp. 415423, 2003.

[19] Y. Schwitalle, M. Linnebacher, E. Ripberger, J. Gebert, and M. von Knebel Doeberitz, "Immunogenic peptides generated by frameshift mutations in DNA mismatch repair-deficient cancer cells," Cancer Immunity, vol. 4, article 14, 2004.

[20] D. Tougeron, E. Fauquembergue, A. Rouquette, et al., "Tumorinfiltrating lymphocytes in colorectal cancers with microsatellite instability are correlated with the number and spectrum of frameshift mutations," Modern Pathology, vol. 22, no. 9, pp. 1186-1195, 2009.

[21] Y. Schwitalle, M. Kloor, S. Eiermann, et al., "Immune response against frameshift-induced neopeptides in HNPCC patients and healthy HNPCC mutation carriers," Gastroenterology, vol. 134, no. 4, pp. 988-997, 2008.

[22] R. J. F. Loos and C. Bouchard, "FTO: the first gene contributing to common forms of human obesity," Obesity Reviews, vol. 9, no. 3, pp. 246-250, 2008.

[23] S. M. Woerner, A. Benner, Y. P. Yuan, P. Bork, M. Knebel Doeberitz, and J. F. Gebert, SelTarbase, A Database for Prediction of Selective Targets in MSI-H Tumorigenesis, release 200804, http://www.seltarbase.org/.

[24] T. Ishikawa, T. Fujita, Y. Suzuki, et al., "Tumor-specific immunological recognition of frameshift-mutated peptides in colon cancer with microsatellite instability," Cancer Research, vol. 63, no. 17, pp. 5564-5572, 2003. 


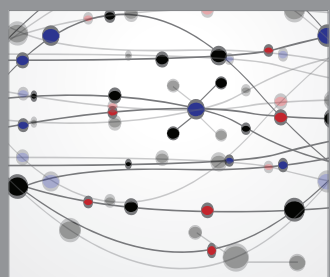

The Scientific World Journal
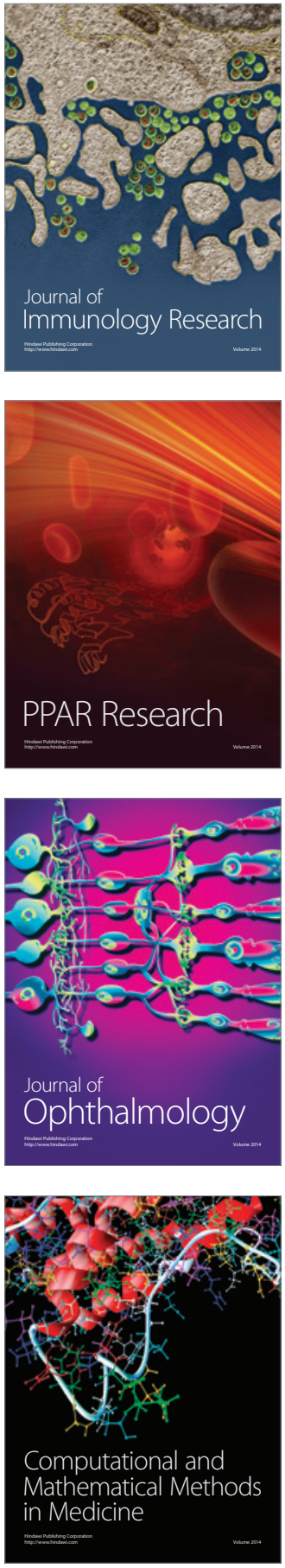

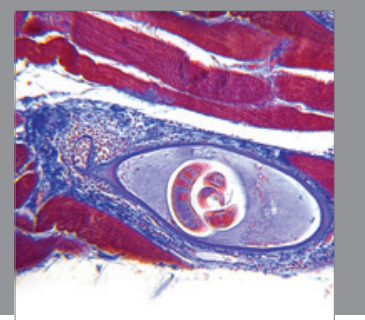

Gastroenterology

Research and Practice
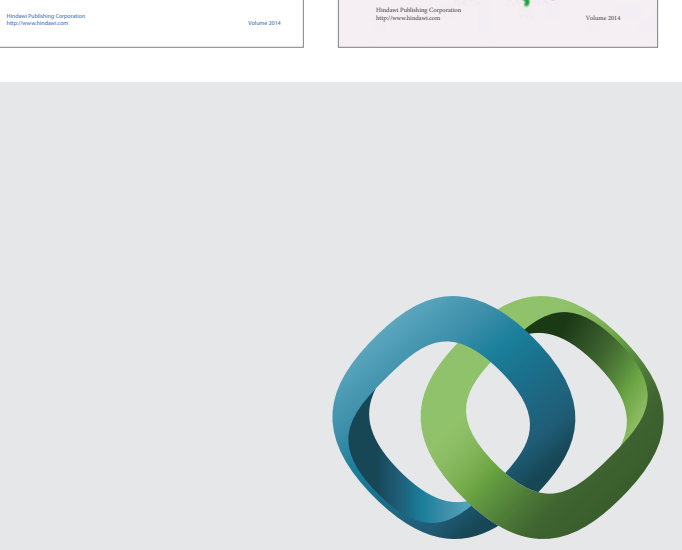

\section{Hindawi}

Submit your manuscripts at

http://www.hindawi.com
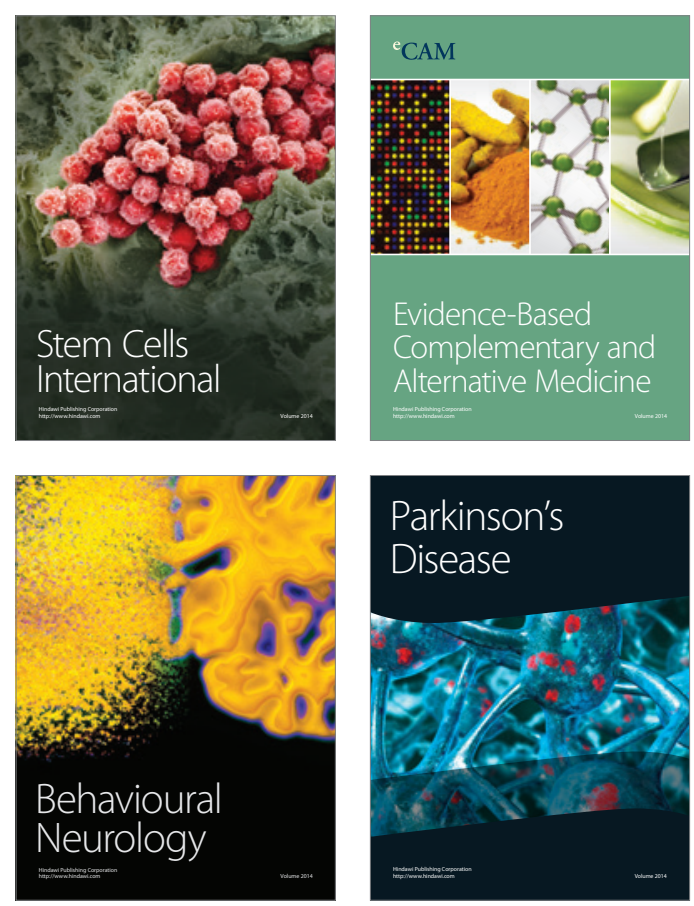

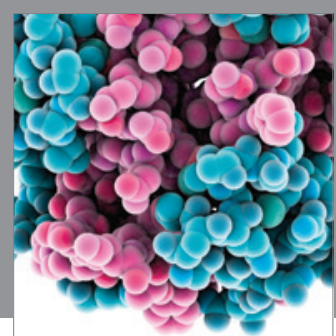

Journal of
Diabetes Research

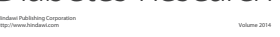

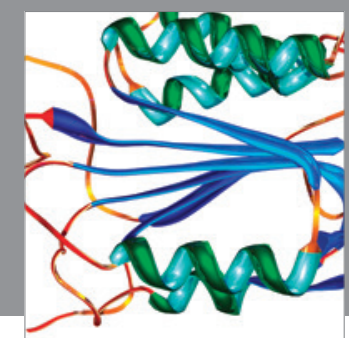

Disease Markers
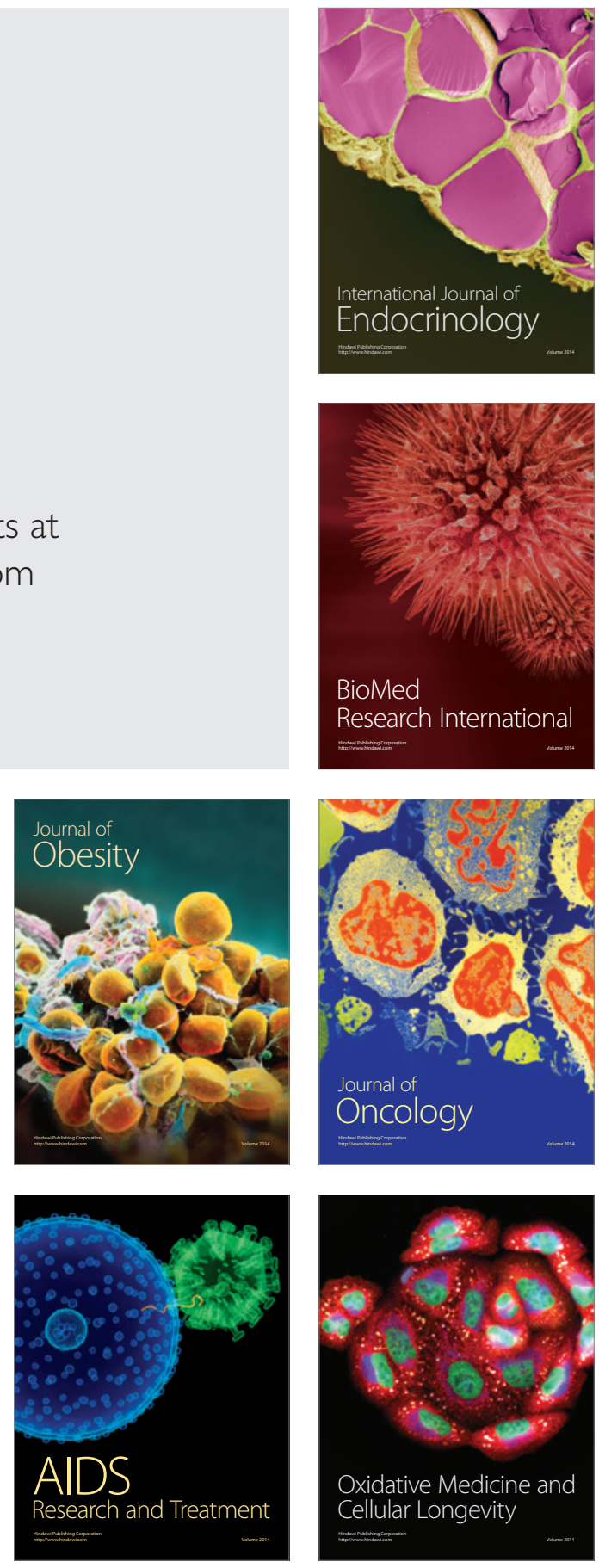